\title{
A Network Pharmacology Approach to Reveal the Underlying Mechanisms of Paeonia lactiflora Pall. On the Treatment of Alzheimer's Disease
}

\author{
Qiang Zeng, ${ }^{1,2,3}$ Longfei Li, ${ }^{4}$ Yu Jin, ${ }^{2,3}$ Zongzheng Chen, ${ }^{1,2,3}$ Lihong Duan,,3 \\ Meiqun Cao $\mathbb{D}^{\mathrm{D}},{ }^{2,3} \mathrm{Min} \mathrm{Ma} \mathbb{D}^{1},{ }^{1}$ and Zhengzhi $\mathrm{Wu} \mathbb{D}^{2,3}$ \\ ${ }^{1}$ Integrated Chinese and Western Medicine Postdoctoral Research Station, School of Traditional Chinese Medicine, \\ Jinan University, Guangzhou 510632, China \\ ${ }^{2}$ The First Affiliated Hospital of Shenzhen University, Shenzhen Second People's Hospital, Shenzhen 518035, China \\ ${ }^{3}$ Shenzhen Institute of Geriatrics, Shenzhen 518020, China \\ ${ }^{4}$ Laboratory of Molecular Pharmacology, Department of Pharmacology, School of Pharmacy, Southwest Medical University, \\ Luzhou 646000, China
}

Correspondence should be addressed to Min Ma; tmamin@jnu.edu.cn and Zhengzhi Wu; szwzz001@163.com

Received 23 May 2019; Revised 23 July 2019; Accepted 13 August 2019; Published 16 November 2019

Academic Editor: Jae Youl Cho

Copyright (C) 2019 Qiang Zeng et al. This is an open access article distributed under the Creative Commons Attribution License, which permits unrestricted use, distribution, and reproduction in any medium, provided the original work is properly cited.

Objective. To investigate the potential active compounds and underlying mechanisms of Paeonia lactiflora Pall. (PLP) on the treatment of Alzheimer's disease (AD) based on network pharmacology. Methods. The active components of PLP were collected from Traditional Chinese Medicine System Pharmacology (TCMSP) database, and their possible target proteins were predicted using TCMSP, SwissTargetPrediction, and STITCH databases. The putative AD-related target proteins were identified from Therapeutic Target Database (TTD), GeneCards, and MalaCards database. The compound-target-disease network interactions were established to obtain the key targets about PLP acting on AD by network topology analysis. Then, the function annotation and signaling pathways of key targets were performed by GO and KEGG enrichment analysis using DAVID tools. Finally, the binding capacity between active ingredients and key targets was validated by molecular docking using SystemsDock tools. Results. There were 7 active compounds involving in 151 predicted targets identified in PLP. Besides, a total of 160 AD-related targets were identified. Among these targets, 30 shared targets of PLP and AD were acquired. After topological analysis of the PLP potential target-AD target network, 33 key targets that were highly responsible for the therapeutic effects of PLP on AD were obtained. Further GO and KEGG enrichment analysis showed that these key targets were significantly involved in multiple biological processes and pathways which participated in cell apoptosis and inflammatory response and maintained the function of neurons to accomplish the anti-AD activity. The molecular docking analysis verified that the 7 active compounds had definite affinity with the key targets. Conclusions. The ameliorative effects of PLP on AD were predicted to be associated with regulating neural cell apoptosis, inflammatory response, and neurotrophy via various pathways such as PI3K-Akt signaling pathway, MAPK signaling pathway, and neurotrophin signaling pathway.

\section{Introduction}

Alzheimer's disease (AD) is one of the commonest neurodegenerative diseases with high incidence and intricate pathogenesis. Unfortunately, there is no efficacious treatment options for $\mathrm{AD}$ patients. AD contributes to about twothirds of dementia cases and affects more than 5 million people in US [1]. The prevalence of $\mathrm{AD}$ is everincreasing with years, and $\mathrm{AD}$ has been the sixth primary cause of death in the US, although the AD-induced death emerges on average 8.5 years [2]. The clinical features of $\mathrm{AD}$ contain memory loss and cognitive impairment at early stage, subsequent topographical difficulties, and alongside loss of confidence, judgement and attention. As the condition 
progresses, cognitive deficiency becomes deteriorative and widespread so as to interfere with activities of daily living and bring poor quality of life to the patients and their families [3]. However, the current mainstream treatments for $\mathrm{AD}$ such as acetylcholinesterase (AChE) inhibitors and $\mathrm{N}$-methyl-D-aspartate (NMDA) receptor antagonists show limited efficacy. The major pathogenic events leading to $\mathrm{AD}$ are attributed to the accumulation of insoluble amyloid- $\beta(A \beta)$ to form senile plaque and aggregation of microtubule protein tau in neurofibrillary tangles (NFTs) in neurons [4]. Recently, the clinical trials showed that new drugs targeting $A \beta$ or tau failed to improve cognitive ability and clinical outcomes of AD patients, and thus they were discontinued. It suggested that the efficacy of singletarget drug was limited and hard to meet clinical needs [5-7]. It is important and necessary to develop novel drugs with multitargets for $\mathrm{AD}$ treatment.

Traditional Chinese Medicines (TCMs) has been used for the treatment of dementia for hundreds of years in China. According to the theory of TCM, AD pertains to long-lasting nutrition deficiency in the brain as a result of obstruction of blood flow and disturbance of "Qi" motion in liver [8]. Paeonia lactiflora Pall. (PLP) is a well-known and widely used herbal medicine that can nourish blood and regulate the liver. Modern pharmacological studies disclosed that formulae containing PLP had evident activities in reducing tau aggregation and ameliorating cognition deficits [9-11]. The aqueous and ethanol extracts of PLP exhibited strong inhibition on $\mathrm{AChE}$ activity as indicated by $\mathrm{IC}_{50}$ values at 20 and $8 \mu \mathrm{g} / \mathrm{ml}$, which was even stronger than Salvia miltiorrhiza Bge. and Polygonum multijiorum Thunb., implying great potential for AD treatment [12]. Although paeoniflorin was reported to be one of the active ingredients in PLP which could improve dementia through neuroprotection and anti-inflammation, the other active compounds in PLP and possible multitarget mechanisms were seldom reported $[13,14]$.

Network pharmacology is an emerging approach for drug discovery. Up to date, this approach has been successfully employed to elucidate the multitarget effects of TCM, which is consistent with the holistic perspective of TCM theory. The use of network pharmacology in the research of TCM integrates phytochemistry, pharmacology, and bioinformatics; effectively bridges the gap between western medicine and traditional medicine; and also greatly facilitates mechanistic studies on the synergistic actions of TCM [15]. In the current study, the active compounds and underlying mechanisms of PLP acting on $\mathrm{AD}$ were comprehensively investigated using the network pharmacology approach. The compounds in PLP and their putative target proteins were identified from the public databases. The biological processes and underlying pathways associated with PLP acting on AD were obtained by enrichment analysis. The combination between the active ingredients in PLP and the key targets was evaluated by simulative molecular docking as well. The present study elucidated the potential active compounds and underlying mechanisms of PLP acting on $\mathrm{AD}$ and provided theoretic evidence for the multi-ingredient and multitarget effects of PLP, suggesting the feasibility of developing PLP or its active compounds as alternative therapy for $\mathrm{AD}$.

\section{Materials and Methods}

2.1. Identification of Chemical Ingredients in PLP. The chemical ingredients in PLP were identified from Traditional Chinese Medicine System Pharmacology (TCMSP, http:// lsp.nwu.edu.cn/browse.php) database [16]. The database provides comprehensive information about ingredients in herbs including chemical structure, oral bioavailability, Caco-2 intestinal epithelial permeability, half-life, drug likeness, drug targets, and their association with diseases and interaction network, etc. The pharmacokinetic properties including absorption, distribution, metabolism, and excretion (ADME) are important contributors for bioactivities of drug. In this study, three ADME-related parameters including oral bioavailability $(\mathrm{OB}) \geq 30 \%$, half-life $(\mathrm{HL}) \geq 4$, and drug likeness (DL) $\geq 0.18$ were employed to identify the potential active ingredients in PLP. As suggested by TCMSP, the compounds with $\mathrm{OB} \geq 30 \%$ and $\mathrm{HL} \geq 4$ have good absorption and slow metabolism after oral administration. The compounds with $\mathrm{DL} \geq 0.18$ were chemically suitable for drug development.

2.2. Prediction of Compound-Related Targets. The compound-related targets were predicted depending on chemical similarities and pharmacophore models via TCMSP, SwissTargetPrediction (http://www.swisstargetprediction.ch/), and STITCH (http://stitch.embl.de/) databases. The probability value of each target marked in SwissTargetPrediction database was used to give a rank list for the targets and evaluate the accuracy of the predictions, whose probability value $\geq 0.5$ was collected in our present study [17]. Besides, confidence score marked in STITCH database provided a reference to define a set of high-confidence interactions between compounds and protein modules [18]. The target proteins with confidence score $\geq 7$ were identified as compound-related targets.

2.3. Identification of AD-Related Targets. The known ADrelated targets were collected from three databases including TTD (Therapeutic Target Database, https://db.idrblab.org/ ttd/), GeneCards (https://www.genecards.org/), and MalaCards (https://www.malacards.org/pages/info).

All the targets obtained above were standardized as gene names and UniProt IDs by searching from UniprotKB (https://www.uniprot.org/) database with "Homo sapiens" species [19].

2.4. Network Construction and Topological Analysis. The compound-target network of PLP and disease-target network of $\mathrm{AD}$, as well as PLP potential target-AD target interaction network, was constructed by Cytoscape v3.7.0 software which is a useful tool for analysis and visualization of the biological network. The topological analysis was performed by the network analyzer module of Cytoscape software. Three topological parameters including degree 
centrality (DC), betweenness centrality (BC), and closeness centrality (CC) were used to estimate the central properties of the nodes in the network. In the PLP potential target-AD target interaction network, $\mathrm{DC} \geq$ median $\mathrm{DC}, \mathrm{BC} \geq$ median $\mathrm{BC}$, and $\mathrm{CC} \geq$ median $\mathrm{CC}$ were employed to screen the key targets of PLP acting on AD.

\subsection{GO and KEGG Pathway Enrichment Analysis.} DAVID (https://david.ncifcrf.gov/) is an online biological knowledgebase and an analytic tool to extract biological information about gene functional classification, functional annotation, and enriched pathways [20]. Gene Ontology (GO) analysis including biological process, cell component, and molecular function, as well as KEGG pathway enrichment analysis, were performed using DAVID database. GO terms with Bonferroni value $<0.05$ and KEGG pathways with $P$ value $<0.05$ were considered to have significance.

2.6. Validation of the Binding Capacity between Active Ingredients and Key Targets by Molecular Docking. Molecular docking is a useful method for drug targets and drug screening research by mimicking the interactions between compounds and proteins to predict their binding capacity and affinity based on their structures. SystemsDock (http://systemsdock.unit.oist.jp/), a web server for assessing protein-ligand binding property, permits high-precision docking simulation and molecular pathway map for comprehensive characterization of ligand selectivity and interpretation of ligand action on a complex molecular network [21]. The crystal structure of the key targets were obtained from PDB (Protein Data Bank) database, and then the molecular docking results between active ingredients in PLP and key targets were analyzed using systemsDock to validate their binding properties. Docking score is a parameter that is the predicted binding affinity to each of target proteins. Docking score at 5.52 is set as the cutoff to classify the good binding capacity between a compound and protein [21].

\section{Results}

3.1. Compound-Target Network of PLP. Total 85 compounds were identified in PLP including terpenoids, glycosides, flavonoids, oleum volatile, and phenols. According to the characteristics of oral bioavailability, half-life, and drug likeness of the compounds, 7 compounds were screened out as the potential active ingredients including $\beta$-sitosterol, kaempferol, lactiflorin, mairin, paeoniflorigenone, paeoniflorin, and palbinone, which are listed in Table 1. The characteristics of the 85 compounds in PLP are shown in Stable 1.

From the TCMSP, SwissTargetPrediction, and STITCH databases, a total of 151 compound-related targets were identified. Among these targets, there were 57 targets involved in $\beta$-sitosterol, 58 targets involved in kaempferol, 5 targets related to lactiflorin, 24 targets related to mairin, 18 targets related to paeoniflorin, 3 targets involved in palbinone, and only one target connected to paeoniflorigenone.
These seven compounds also had some shared bioactive targets and connection networks. The targets of each compound are listed in Stable 2, and the compound-target network of PLP is shown in Figure 1(a).

To clarify the characteristics of compound-related targets on molecular function and pathway level, GO function and KEGG pathway enrichment analysis were performed (Figures 1(b)-1(e)). Most of these potential targets existed on the plasma membrane with molecular function of protein binding. Specifically, these targets were involved in biological processes such as oxidation-reduction process, regulation of transcription, response to drug, signal transduction, and inflammatory response. Furthermore, the results of KEGG enrichment analysis demonstrated that there were totally 101 pathways $(P$ value $<0.05)$ affected by the active ingredients of PLP. The top 10 (count number $\geq 15$ ) enriched pathways contained metabolic pathways, pathways in cancer, hepatitis B, tuberculosis, neuroactive ligand-receptor interaction, nonalcoholic fatty liver disease, toxoplasmosis, steroid hormone biosynthesis, influenza $\mathrm{A}$, and calcium signaling pathway.

3.2. Disease-Target Network of AD. From the TTD, GeneCards, and MalaCards databases, a total of 160 AD-related targets were identified (Stable 3), among which 142 targets showed high interactions (confidence score $\geq 7$ ) in the protein-protein interactions map generated by the STRING database. The disease-target network of $\mathrm{AD}$ was constructed and consisted of 142 nodes (targets with high interactions) and 626 link edges. The central attributes of each node were evaluated by topology analysis. In Figure 2, the size of nodes was proportional to degree centrality. Notably, amyloid precursor protein $($ APP, degree $=56)$ which played an essential role in the pathogenesis of AD brain was identified as the most important protein in this disease-target network. In addition, according to both degree and betweenness centrality, INS, AKT1, IL6, TP53, BDNF, and NGF were also recognized as important $\mathrm{AD}$ targets.

3.3. PLP Potential Target-AD Target Network. To identify the relationship between the PLP potential targets and AD-related targets, their targets were analyzed, and thus 30 shared targets of PLP and AD were acquired. The interaction network of PLP potential target-AD target was established and shown in Figure 3(a). There were 260 targets and 1634 link edges which had high interactions with confidence score $\geq 7$ in the STRING database. Based on criteria of $\mathrm{DC} \geq$ median $\mathrm{DC}, \mathrm{BC} \geq$ median $\mathrm{BC}$, and $\mathrm{CC} \geq$ median $\mathrm{CC}$, 33 key targets of PLP acting on AD were obtained (Table 2) and further used to construct the interaction network consisting of 33 nodes and 254 link edges (Figure 3(b)).

These key targets were primarily distributed in cellular components such as cytosol and cytoplasm and mainly involved in biological processes such as regulation of transcription, regulation of apoptotic process, regulation of gene expression, inflammatory response, and regulation of nitric oxide biosynthetic process via molecular function of protein binding by GO enrichment analysis (Figure 3(c)). 
TABLe 1: The characteristics of active ingredients in PLP.

\begin{tabular}{|c|c|c|c|c|c|c|}
\hline Compounds & Molecular formula & Structure & Molecular weight & OB (\%) & $\mathrm{HL}$ & $\overline{\mathrm{DL}}$ \\
\hline$\beta$-sitosterol & $\mathrm{C}_{29} \mathrm{H}_{50} \mathrm{O}$ & & 414.79 & 36.91 & 5.36 & 0.75 \\
\hline Kaempferol & $\mathrm{C}_{15} \mathrm{H}_{10} \mathrm{O}_{6}$ & & 286.25 & 41.88 & 14.74 & 0.24 \\
\hline
\end{tabular}

Lactiflorin

Mairin $\quad \mathrm{C}_{30} \mathrm{H}_{48} \mathrm{O}_{3}$

Paeoniflorigenone

Paeoniflorin

Palbinone




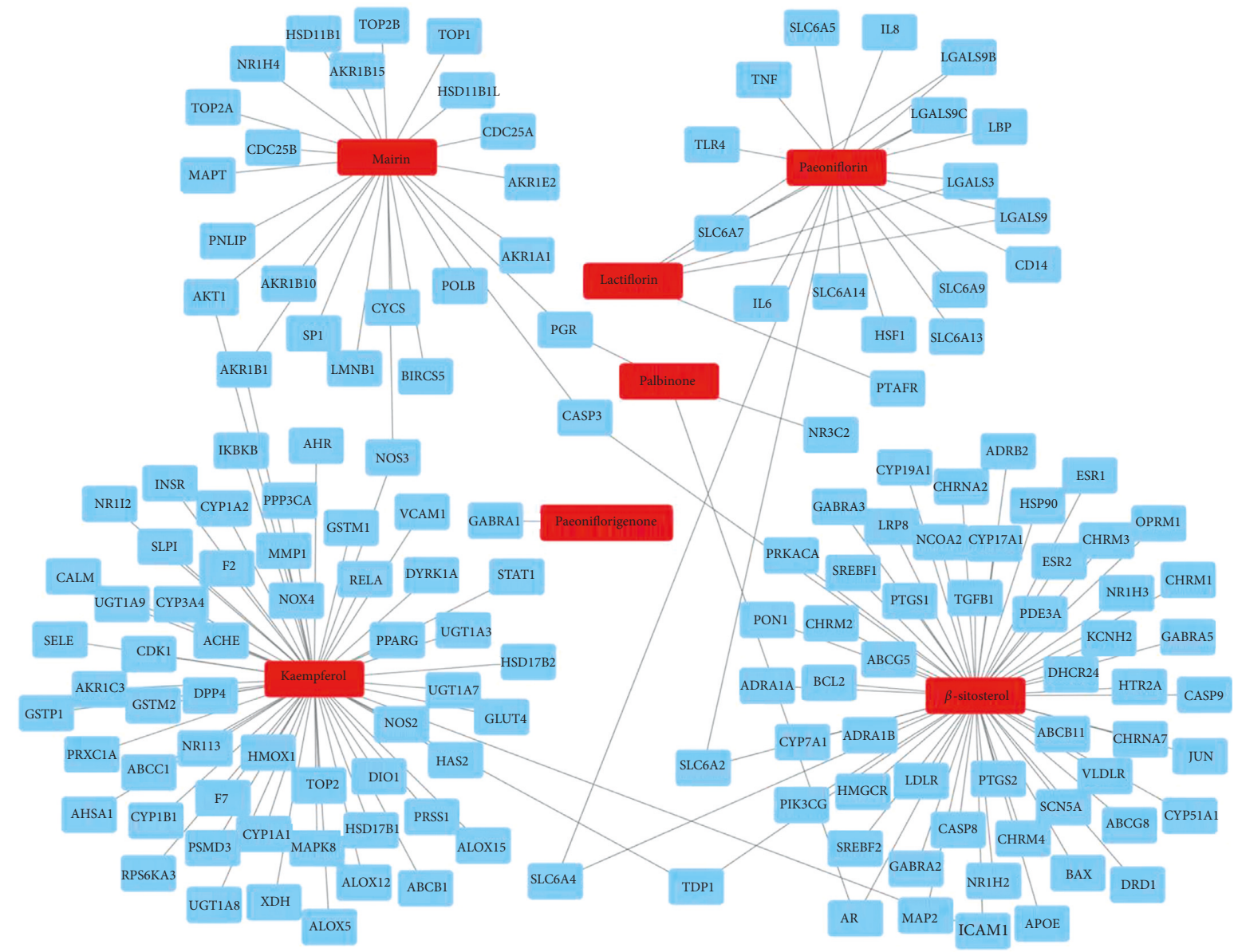

(a)

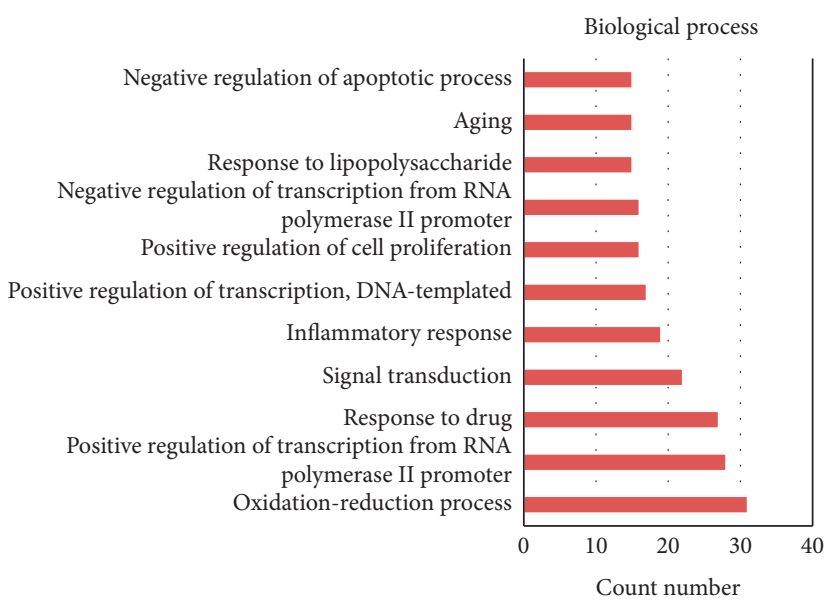

(b)

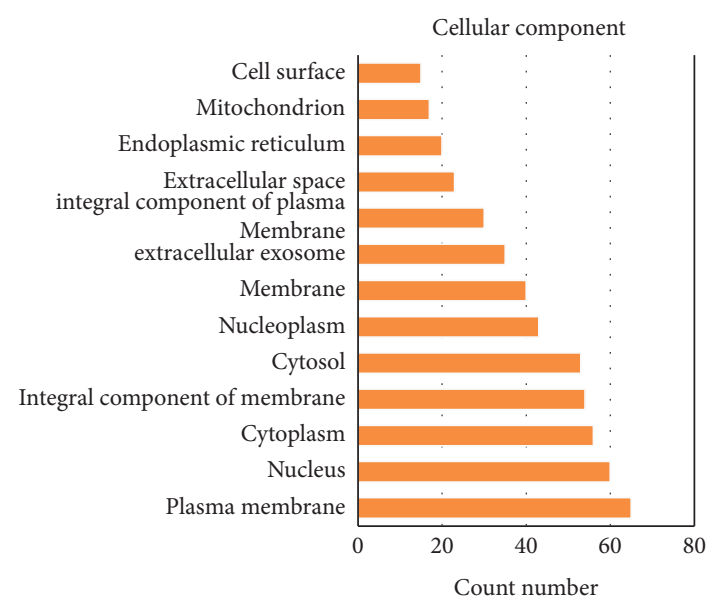

(c)

Figure 1: Continued. 


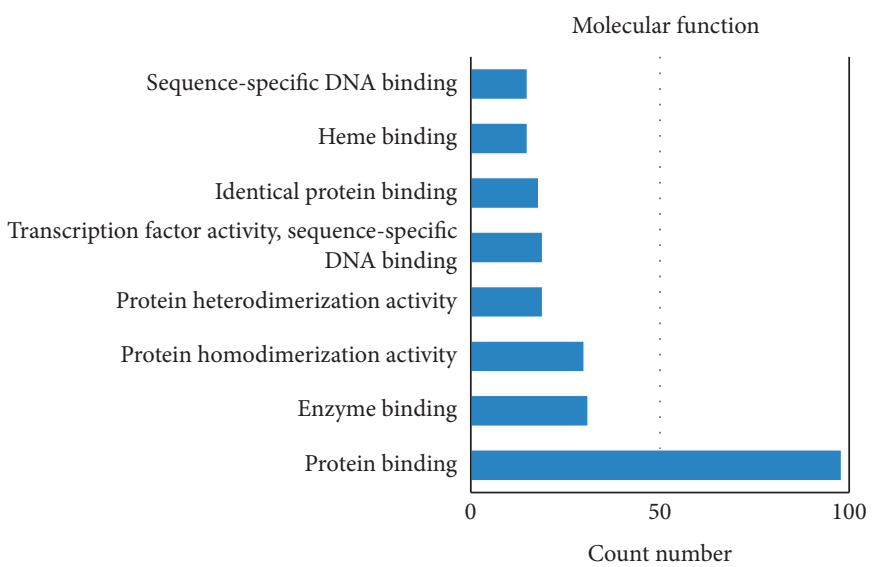

(d)

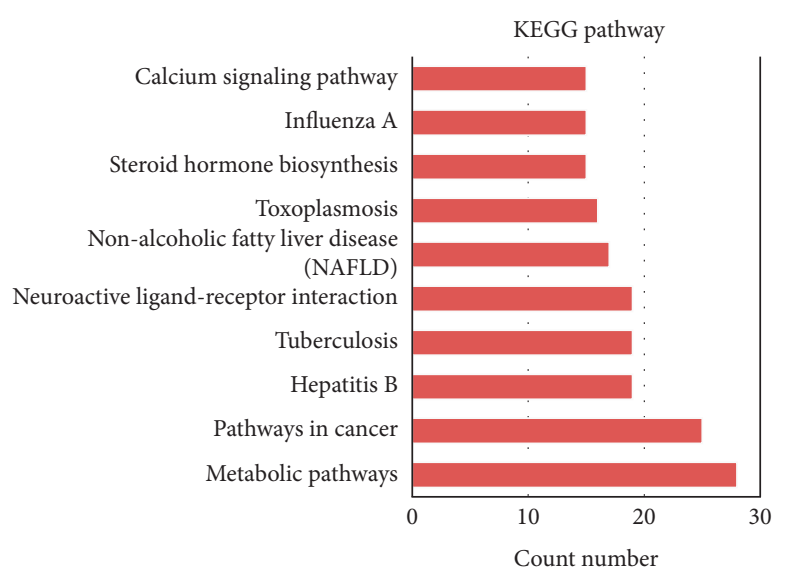

(e)

Figure 1: (a) Compound-target network of PLP. Red rectangle nodes represent potential active compounds in PLP, while blue rectangle nodes represent potential targets of PLP. (b)-(d) GO enrichment analysis for potential targets of PLP (count number $\geq 15$ ). (e) KEGG enrichment analysis for potential targets of PLP (count number $\geq 15$ ).

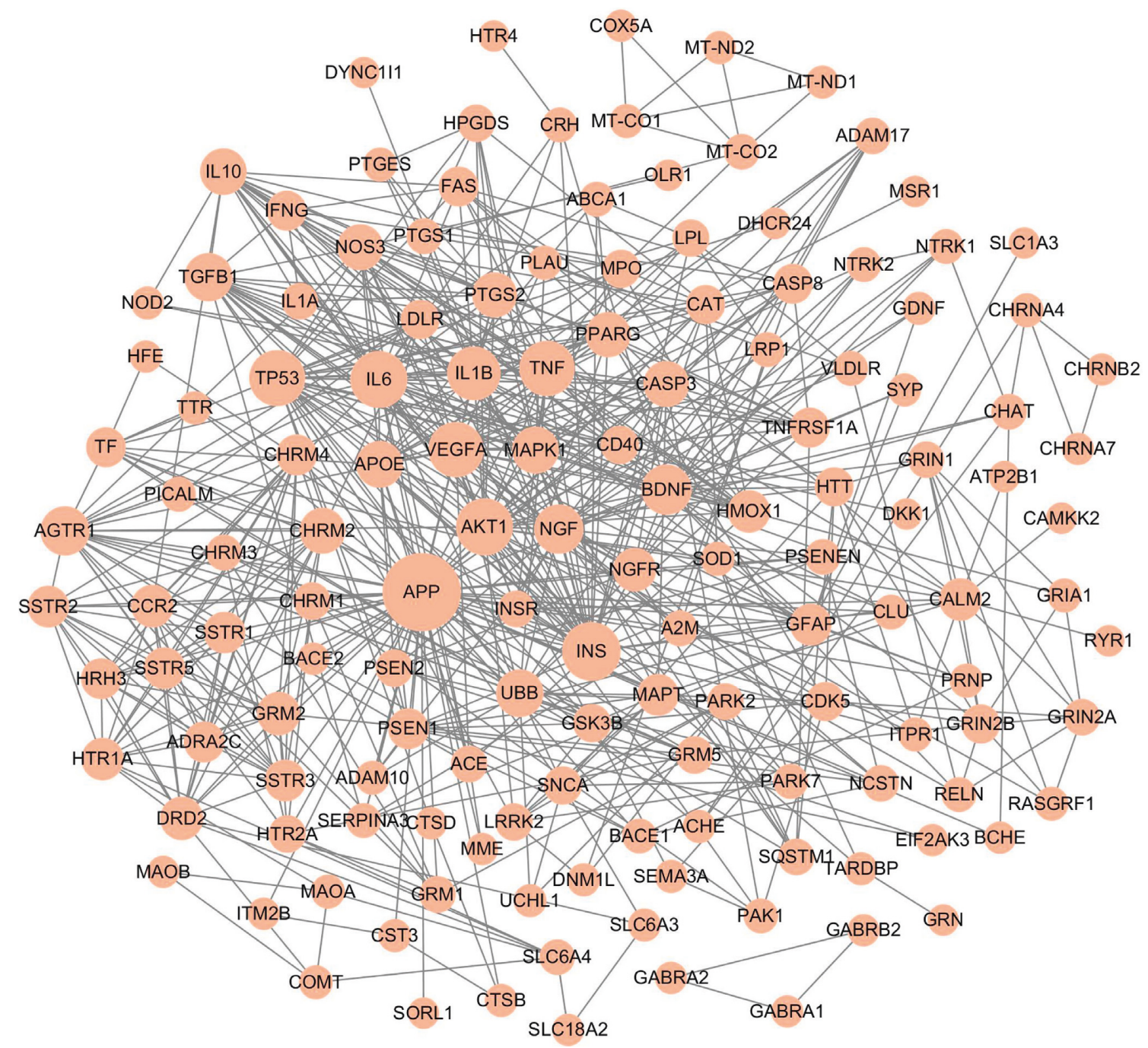

Figure 2: Disease-target network of AD. Pink ellipse nodes represent AD-related targets, and the size of nodes is proportional to degree centrality by topology analysis. 


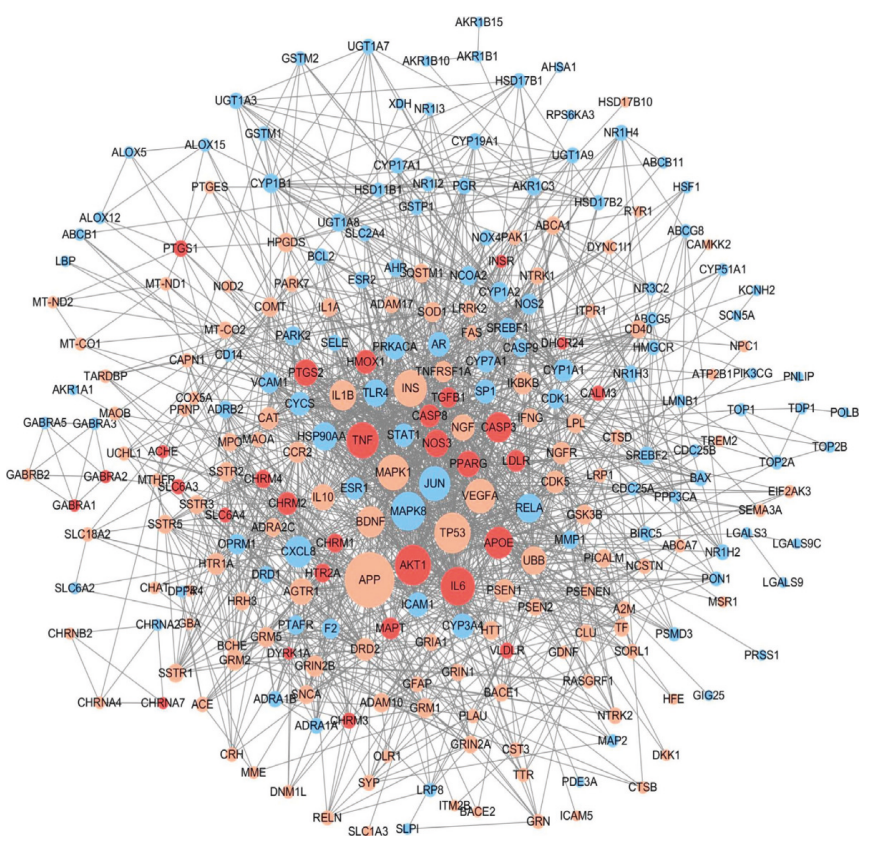

(a)

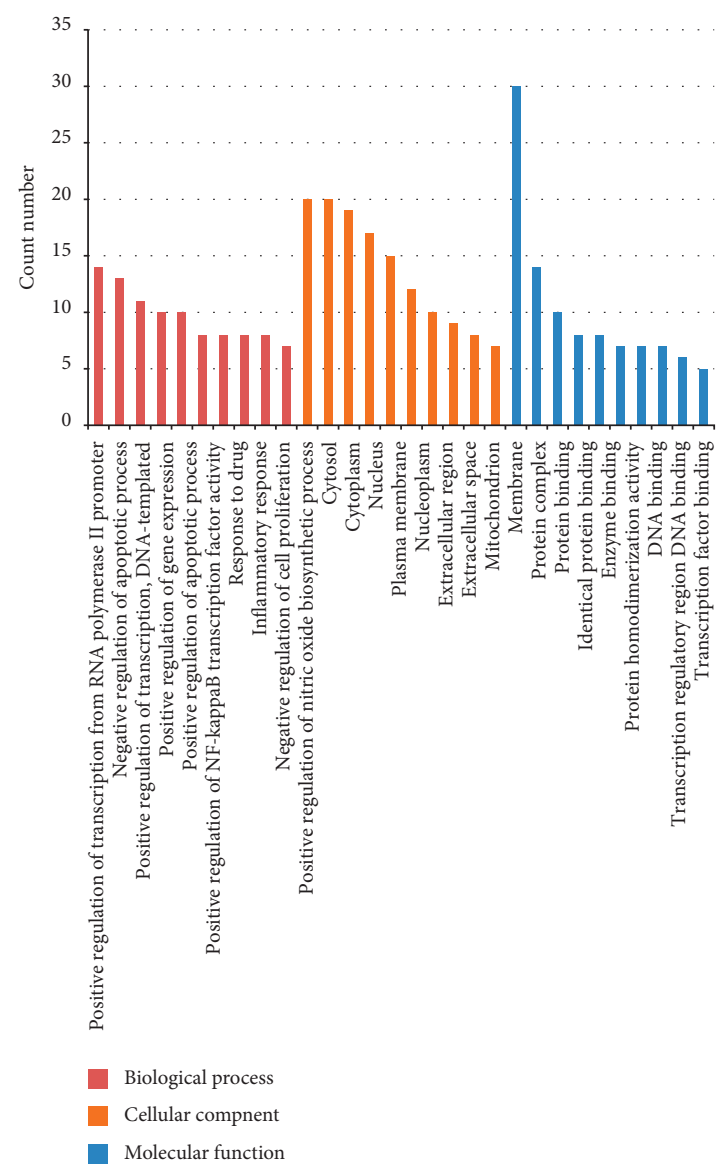

(c)

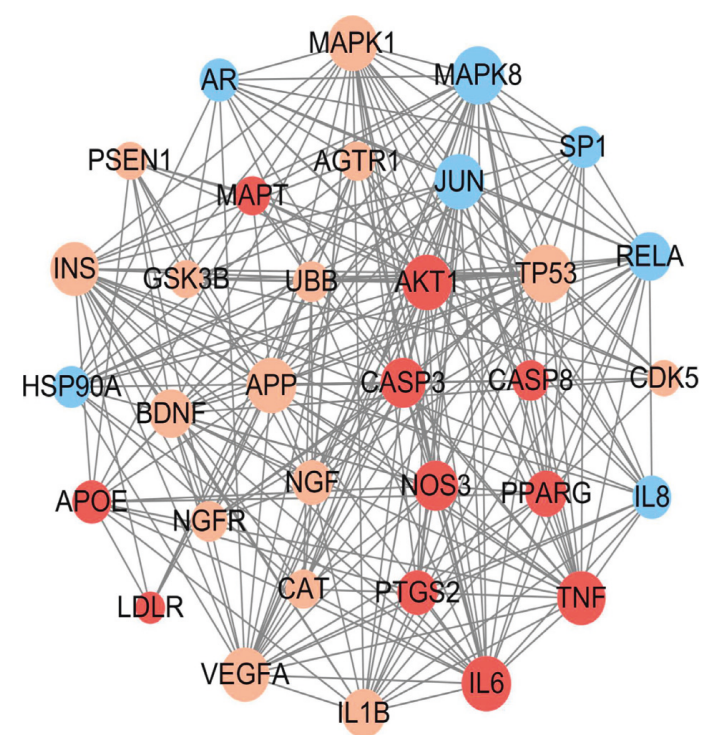

(b)

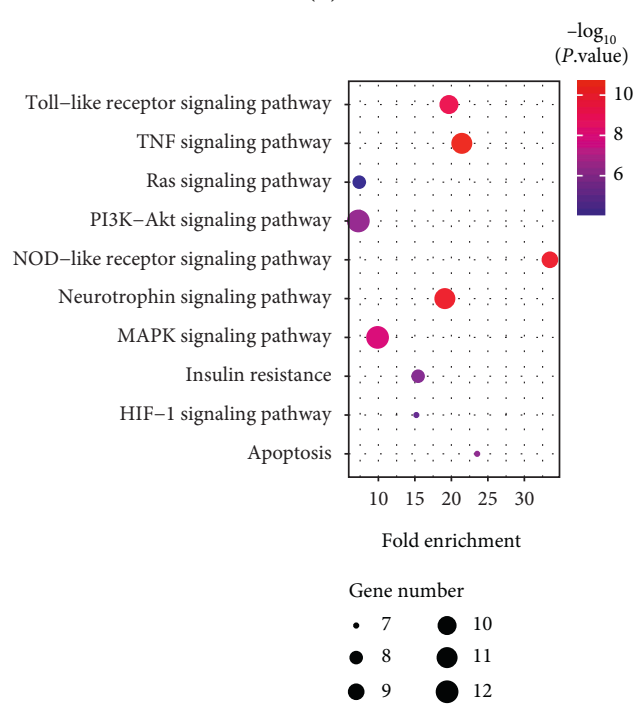

(d)

Figure 3: (a) PLP potential target-AD target network. (b) Network of the 33 key targets. Blue ellipse nodes stand for PLP potential targets, pink ellipse nodes represent AD-related targets, and red ellipse nodes represent the shared targets of PLP and AD. The size of nodes is proportional to degree centrality by topology analysis. (c) GO enrichment analysis for 33 key targets. (d) KEGG enrichment analysis for 33 key targets. 
TABLe 2: The 33 key targets of PLP acting on AD.

\begin{tabular}{|c|c|c|c|c|}
\hline Target & Name & Degree & Betweenness centrality & Closeness centrality \\
\hline APP & Amyloid precursor protein & 81 & 0.192 & 0.517 \\
\hline TP53 & Tumor protein $\mathrm{P} 53$ & 54 & 0.096 & 0.507 \\
\hline $\mathrm{AKT1}$ & RAC-alpha serine/threonine-protein kinase & 54 & 0.039 & 0.483 \\
\hline MAPK8 & Mitogen-activated protein kinase 8 & 51 & 0.042 & 0.489 \\
\hline IL6 & Interleukin 6 & 50 & 0.035 & 0.485 \\
\hline INS & Insulin & 47 & 0.060 & 0.490 \\
\hline TNF & Tumor necrosis factor & 46 & 0.022 & 0.473 \\
\hline MAPK1 & Mitogen-activated protein kinase 1 & 45 & 0.036 & 0.481 \\
\hline JUN & Transcription factor AP1 & 44 & 0.026 & 0.482 \\
\hline VEGFA & Vascular endothelial growth factor A & 43 & 0.029 & 0.475 \\
\hline APOE & Apolipoprotein E & 38 & 0.040 & 0.458 \\
\hline IL8 & Interleukin 8 & 38 & 0.028 & 0.444 \\
\hline $\mathrm{BDNF}$ & Brain-derived neurotrophic factor & 37 & 0.039 & 0.465 \\
\hline CASP3 & Caspase 3 & 35 & 0.023 & 0.461 \\
\hline IL1B & Interleukin- $1 \beta$ & 35 & 0.016 & 0.443 \\
\hline RELA & Transcription factor p65 & 33 & 0.012 & 0.456 \\
\hline UBB & Ubiquitin $\mathrm{B}$ & 32 & 0.032 & 0.441 \\
\hline HSP90A & Heat shock protein HSP 90 & 32 & 0.031 & 0.431 \\
\hline NOS3 & Endothelial nitric oxide synthase & 30 & 0.018 & 0.449 \\
\hline AGTR1 & Type-1 angiotensin II receptor & 29 & 0.016 & 0.437 \\
\hline PTGS2 & Prostaglandin $\mathrm{G} / \mathrm{H}$ synthase 2 & 29 & 0.026 & 0.435 \\
\hline NGF & Nerve growth factor & 28 & 0.013 & 0.443 \\
\hline PPARG & Peroxisome proliferator activated receptor $\gamma$ & 28 & 0.013 & 0.434 \\
\hline $\mathrm{AR}$ & Androgen receptor & 25 & 0.026 & 0.421 \\
\hline SP1 & Sp1 transcription factor & 24 & 0.014 & 0.424 \\
\hline CASP8 & Caspase 8 & 22 & 0.007 & 0.436 \\
\hline PSEN1 & Presenilin 1 & 22 & 0.015 & 0.427 \\
\hline CDK5 & Cyclin-dependent kinase 5 & 22 & 0.013 & 0.424 \\
\hline NGFR & Nerve growth factor receptor & 21 & 0.011 & 0.440 \\
\hline CAT & Catalase & 21 & 0.020 & 0.423 \\
\hline MAPT & Microtubule associated protein tau & 20 & 0.010 & 0.422 \\
\hline LDLR & Low-density lipoprotein receptor & 20 & 0.020 & 0.417 \\
\hline GSK3B & Glycogen synthase kinase- $3 \beta$ & 17 & 0.006 & 0.433 \\
\hline
\end{tabular}

The KEGG enrichment analysis was carried out to further explore the underlying mechanisms of PLP on AD. The representative top 10 pathways based on the number of enriched genes as well as fold changes and $P$ value are shown in Figure 3(d). These key targets were closely related to PI3KAkt signaling pathway, MAPK signaling pathway, neurotrophin signaling pathway, TNF signaling pathway, toll-like receptor signaling pathway, etc., which participated in cell apoptosis and inflammatory response and maintained the function of neurons to accomplish the anti-AD activity of PLP.

\subsection{The Binding Capacity between Active Compounds and Key} Targets by Molecular Docking. To further verify the binding capacity between active compounds and key targets, molecular docking through systemsDock was performed. The docking results are shown in Figure 4 based on the docking score which was a negative logarithm of the experimental dissociation/inhibition constant, ranging from 0 to 10 that represented weak to strong binding. The docking scores showed that the active compounds of PLP, especially $\beta$-sitosterol and mairin, had good binding activity to $\mathrm{AD}$ putative targets included in the key targets, indicating the specific action proteins of PLP for AD treatment.

\section{Discussion}

According to the theory of TCM, long-term nutrition deficiency in the brain as a result of blood stasis and "Qi" stagnation in liver is one of the pathogenic reasons for $\mathrm{AD}$. PLP is a well-known herbal medicine with a function of nourishing and regulating blood and is commonly used to improve nutrition deficiency in the brain. Among the large numbers of formulae for treatment of dementia, PLP is one of the most widely used herbs and usually works as a monarch herb in a prescription [22]. The present study aimed to explore the potential active compounds and underlying mechanisms of PLP acting on $\mathrm{AD}$ and provided theoretic evidence for developing PLP or its active compounds as alternative therapy for $\mathrm{AD}$. The representative compounds and potential mechanisms of PLP on AD treatment are depicted in Figure 5. In the current study, there were 7 compounds including $\beta$-sitosterol, kaempferol, lactiflorin, mairin, paeoniflorigenone, paeoniflorin, and palbinone identified as the potential active ingredients of PLP, of which the biological activities against AD were reported previously. For example, kaempferol, that is, a natural acetylcholinesterase inhibitor could delay the loss of climbing ability, ameliorate memory deficiency, and reduce oxidative stress and neuroinflammation both in the 


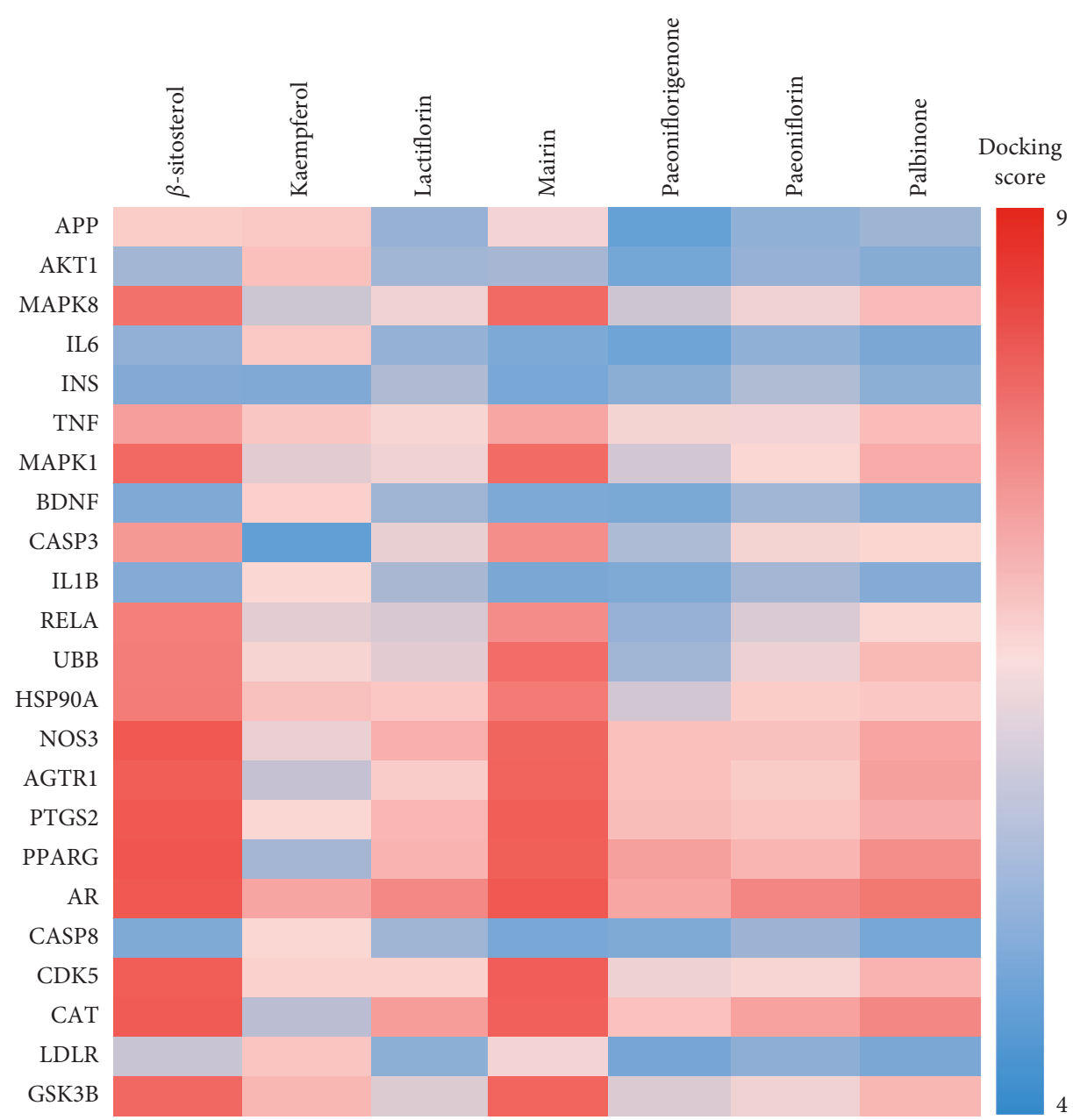

FIGURE 4: Heat map of binding capacity between the active compounds and key targets by molecular docking. The heat map was depicted based on docking scores.

ovariectomized rat model and transgenic Drosophila model of $\mathrm{AD}$ [23-25]. Mairin, also named as betulinic acid, that is, a pentacyclic triterpenoid, could prevent $A \beta /$ streptozotocininduced spatial and passive avoidance memory deficits and reduce $\mathrm{A} \beta$ fibril plaques in the hippocampus region of the $\mathrm{AD}$ rat model through protecting microcirculation, alleviating inflammation, and upregulating BDNF expression $[26,27]$. Paeoniflorin is reported to improve memory deficits, attenuate amyloidogenesis, prevent $\mathrm{A} \beta$-induced astrocytes and microglia activation, and suppress inflammatory responses in the transgenic AD model [14]. $\beta$-sitosterol belongs to the group of phytosterols which are active ingredients existing in a diversity of natural plants. It is reported that $\beta$-sitosterol bound to the active sites of AChE and BChE as an inhibitor by molecular docking and exhibited an $\mathrm{IC}_{50}$ value of 55 and $50 \mu \mathrm{g} / \mathrm{ml}$ against these two enzyme activities [28]. In addition, administration of $\beta$-sitosterol at $10 \mathrm{mg} / \mathrm{kg}$ body weight/day demonstrated gradual improvement in memory deficiency and motor coordination in the transgenic AD model [28]. Therefore, the above active components indicate the effectiveness and diversity of chemical ingredients in PLP for treating AD.
The results of PLP potential target-AD target network analysis acquired 33 key targets of PLP acting on AD. These key targets were mainly involved in biological processes such as regulation of transcription, regulation of apoptotic process, regulation of gene expression, inflammatory response, and regulation of nitric oxide biosynthetic process via molecular function of protein binding. The predicted results in our current study were consistent with some previous publications. It is reported that $\beta$-sitosterol attenuated $\mathrm{A} \beta$ induced neural cell apoptosis and inflammation through downregulating iNOS expression and NF- $\kappa$ B, p38, and ERK activation [29]. Kaempferol inhibited inflammatory response and iNOS expression as well as NO generation through suppressing NF- $\kappa \mathrm{B}, \mathrm{p} 38$, JNK, and AKT phosphorylation [30]. Mairin promoted M2 phenotype microglial polarization and prevented M1 polarization through calmodulin-dependent protein kinase kinase $\beta / \mathrm{AMPK}$ activation, specifically decreased iNOS and TNF- $\alpha$ expression when the BV2 cells were treated with lipopolysaccharide (LPS) [31]. Further molecular docking assay in this study showed that $\beta$-sitosterol and mairin were conferred strong binding activity with MAPK1 (ERK2), MAPK8 (JNK1), 


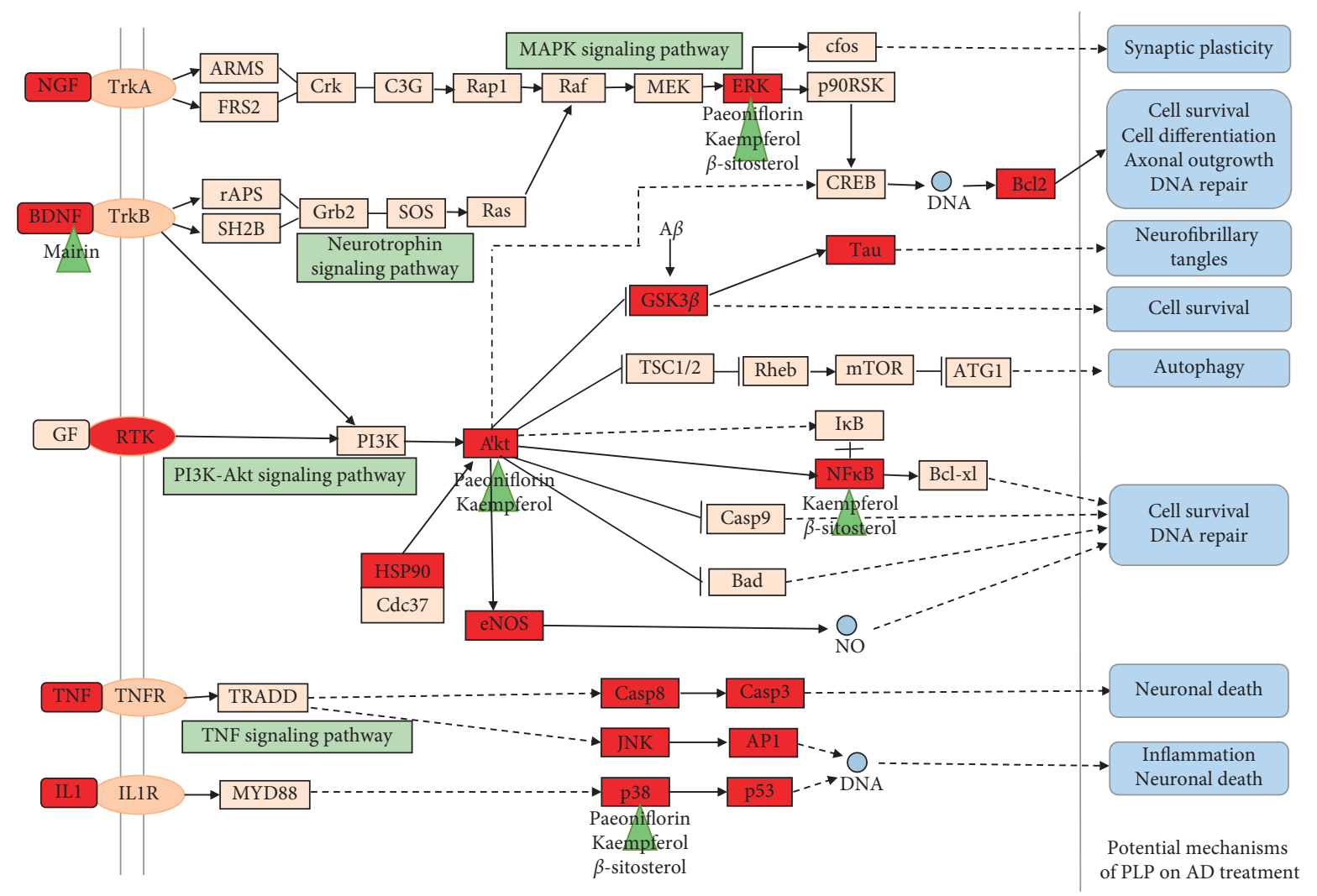

FIgURE 5: Representative compounds and potential mechanisms of PLP on AD treatment. The green triangle stands for representative compounds in PLP, and the red rectangle stands for the compound-related targets.

NOS3, and TNF- $\alpha$, while kaempferol had strong combination with AKT1 and TNF- $\alpha$.

As observed in the results of KEGG pathway enrichment analysis, the key targets of PLP acting on AD were mainly related to PI3K-Akt signaling pathway, MAPK signaling pathway, neurotrophin signaling pathway, TNF signaling pathway, and so on. $\mathrm{AD}$ is an intricate neurodegenerative disease that the underlying mechanisms have not been clearly elucidated. It is recognized that the pathogenesis of $\mathrm{AD}$ is associated with various biological processes such as synaptic loss, specific neurotransmitters reduction, neuroinflammation, and neuronal death [32]. The neural cell death induced by aggregated $\mathrm{A} \beta$ plaque plays a crucial role in the pathogenesis of AD. The PI3K-Akt pathway participates in cell survival and death, particularly exhibiting beneficial effect on cell survival and inhibitory effect on cell apoptosis once Akt is activated [33]. In addition, PI3K-Akt pathway is involved in the initiation of the autophagic process which is a major intracellular machinery for degrading misfolded proteins and damaged organelles and has been reported to be involved in the pathogenesis of AD through acting on its downstream mTOR complex [34]. Notably, ubiquitinproteasome system (UPS) is another major intracellular abnormal protein degradation system in eukaryotic cells that is likely associated with the etiology of $\mathrm{AD}$. Ubiquitin possesses the function of labeling and binding to the proteins for degradation such as APP and $\gamma$-secretase activating protein that contributes to etiology of $\mathrm{AD}[35,36]$. It is found that aberrant form of this protein originating from misreading of UBB gene was accumulated in brain tissues of AD patients [37]. The molecular docking assay in this study showed that $\beta$-sitosterol, mairin, and palbinone presumably bound to UBB with strong affinity, implying the possible target of PLP on AD treatment.

MAPK pathway, the downstream signaling of multiple pathways, participated in synapse plasticity, neural cell survival, cell apoptosis, and neuroinflammation. A lot of studies have demonstrated that the crucial proteins of the MAPK signaling pathway such as ERK1, JNK, and p38 were all elevated in AD animal models, thus targeting these proteins could reduce $\mathrm{A} \beta$ production, tau phosphorylation, neuroinflammation, and synaptic loss, as well as slowed down the degeneration of cognitive function [38-40]. For example, Neurotropin ${ }^{\circledR}$ alleviated the accumulation of $\mathrm{A} \beta$ plaques and $\mathrm{A} \beta$-induced neural cell death via suppression of HIF- $1 \alpha$, p-ERK1/2, p-JNK, and p-p38 in APP/PS1 mice [41]. Therefore, targeting MAPK signaling pathway-related proteins was a promising strategy for $\mathrm{AD}$ treatment.

BDNF and NGF are two of the pivotal factors involved in neurotrophin signaling pathway and play important roles in cholinergic synapse and synaptic plasticity. BDNF contributes to the development of hippocampal structure and function, while NGF promotes the function of cerebrum cholinergic neurons and prohibits neural cell death [42]. It is found that BDNF levels in peripheral blood and in cerebrospinal fluid as well as in hippocampus and neocortex of 
$\mathrm{AD}$ patients were significantly decreased compared with controls. However, NGF level in blood did not show evident change, but NGF levels in cerebrospinal fluid and hippocampus and neocortex of $\mathrm{AD}$ patients were significantly increased, suggesting that aberrations of neurotrophic factors were involved in the etiology and pathogenesis of $\mathrm{AD}$ [43]. Targeting neurotrophin signaling pathway to restore neural function was potential strategy for AD treatment [44]. The results of network analysis in this study also demonstrated that BDNF was one of the key targets related to the treatment of PLP for AD, and kaempferol had strong binding activity with BDNF by molecular docking.

In view of the pathological complexity of disease, herbal medicine can act on various molecules and targets to exert systematic actions on the disease. Network pharmacology is a powerful method to study the synergistic actions and underlying mechanisms of traditional medicines. However, it is a predicting method based on database analysis to find the possible mechanisms of drugs. Further biological studies are warranted to verify the above findings.

\section{Conclusions}

In conclusion, the present study discovered compoundtarget-disease interactions and possible mechanisms of PLP on $\mathrm{AD}$ treatment using network pharmacology strategy and predicted that $\beta$-sitosterol, kaempferol, lactiflorin, mairin, paeoniflorigenone, paeoniflorin, and palbinone were potential active ingredients of PLP, which possibly prevented $A D$ via inhibiting neural cell apoptosis, inflammatory response, and promoting neurotrophy. It provided the theoretic elucidation of the ameliorative effect of PLP against $\mathrm{AD}$ and might facilitate the development of PLP or its active compounds as alternative therapy for $\mathrm{AD}$.

\section{Data Availability}

The data used to support the findings of this study are available from the corresponding author upon request.

\section{Conflicts of Interest}

There are no conflicts of interest to declare.

\section{Authors' Contributions}

$\mathrm{ZW}, \mathrm{MM}$, and MC designed the study and supervised the project. QZ and LL performed the network pharmacology analysis and prepared the manuscript. YJ and ZC collected the data. $\mathrm{LD}, \mathrm{MC}, \mathrm{MM}$, and $\mathrm{ZW}$ all made contributions to the revision of manuscript. All authors read and approved the final manuscript.

\section{Acknowledgments}

This study was financially supported by the National Natural Science Foundation of China (grant no. 81574038), Project of Traditional Chinese Medicine Bureau of Guangdong Province (no. 20191286), Shenzhen Basic Discipline Layout Project (nos. JCYJ20170412161254416 and JCYJ20170413161352000), and Shenzhen Sanming Project of Medicine and Health (no. SZSM201612049).

\section{Supplementary Materials}

The characteristics of 85 compounds in PLP are shown in Supplementary Table 1 . The predicted targets of $\beta$-sitosterol, kaempferol, lactiflorin, mairin, paeoniflorin, palbinone, and paeoniflorigenone are listed in Supplementary Table 2. The acknowledged targets of Alzheimer disease are displayed in Supplementary Table 3. (Supplementary Materials)

\section{References}

[1] R. A. Corriveau, W. J. Koroshetz, J. T. Gladman et al., "Alzheimer's disease-related dementias summit 2016: national research priorities," Neurology, vol. 89, no. 23, pp. 2381-2391, 2017.

[2] Alzheimer's Association, "Alzheimer's disease facts and figures," Alzheimer's \& Dementia, vol. 14, no. 3, pp. 367-429, 2018.

[3] C. A. Lane, J. Hardy, and J. M. Schott, "Alzheimer's disease," European Journal of Neurology, vol. 25, no. 1, pp. 59-70, 2018.

[4] Q. Zeng, W. Siu, L. Li et al., "Autophagy in Alzheimer's disease and promising modulatory effects of herbal medicine," Experimental Gerontology, vol. 119, pp. 100-110, 2019.

[5] R. S. Doody, R. G. Thomas, M. Farlow et al., "Phase 3 trials of solanezumab for mild-to-moderate Alzheimer's disease," New England Journal of Medicine, vol. 370, no. 4, pp. 311-321, 2014.

[6] S. Salloway, R. Sperling, N. C. Fox et al., "Two phase 3 trials of bapineuzumab in mild-to-moderate Alzheimer's disease," New England Journal of Medicine, vol. 370, no. 4, pp. 322-333, 2014.

[7] F. Panza, V. Solfrizzi, D. Seripa et al., "Tau-based therapeutics for Alzheimer's disease: active and passive immunotherapy," Immunotherapy, vol. 8, no. 9, pp. 1119-1134, 2016.

[8] Z. Lin, J. Gu, J. Xiu et al., "Traditional Chinese medicine for senile dementia," Evidence-Based Complementary and Alternative Medicine, vol. 2012, Article ID 692621, 13 pages, 2012.

[9] I. C. Chen, T.-H. Lin, Y.-H. Hsieh et al., "Formulated Chinese medicine Shaoyao-Gancao-Tang reduces tau aggregation and exerts neuroprotection through anti-oxidation and anti-inflammation," Oxidative Medicine and Cellular Longevity, vol. 2018, Article ID 9595741, 16 pages, 2018.

[10] Y. Huang, Z.-Y. Hu, H. Yuan et al., "Danggui-Shaoyao-San improves learning and memory in female SAMP8 via modulation of estradiol," Evidence-Based Complementary and Alternative Medicine, vol. 2014, Article ID 327294, 9 pages, 2014.

[11] Z. Lan, J. Liu, L. Chen et al., "Danggui-Shaoyao-San ameliorates cognition deficits and attenuates oxidative stress-related neuronal apoptosis in d-galactose-induced senescent mice," Journal of Ethnopharmacology, vol. 141, no. 1, pp. 386-395, 2012.

[12] H. Q. Lin, M. T. Ho, L. S. Lau, K. K. Wong, P. C. Shaw, and D. C. C. Wan, "Anti-acetylcholinesterase activities of traditional Chinese medicine for treating Alzheimer's disease," Chemico-Biological Interactions, vol. 175, no. 1-3, pp. 352354, 2008.

[13] X.-H. Ma, W.-J. Duan, Y.-S. Mo et al., "Neuroprotective effect of paeoniflorin on okadaic acid-induced tau hyperphosphorylation 
via calpain/Akt/GSK-3 $\beta$ pathway in SH-SY5Y cells," Brain Research, vol. 1690, pp. 1-11, 2018.

[14] H.-R. Zhang, J.-H. Peng, X.-B. Cheng, B.-Z. Shi, M.-Y. Zhang, and R.-X. Xu, "Paeoniflorin atttenuates amyloidogenesis and the inflammatory responses in a transgenic mouse model of Alzheimer's disease," Neurochemical Research, vol. 40, no. 8, pp. 1583-1592, 2015.

[15] H. Yuan, Q. Ma, H. Cui et al., "How can synergism of traditional medicines benefit from network pharmacology?" Molecules, vol. 22, no. 7, pp. 1135-1153, 2017.

[16] J. Ru, L. Peng, W. Jinan et al., "TCMSP: a database of systems pharmacology for drug discovery from herbal medicines," Journal of Cheminformatics, vol. 6, no. 1, p. 13, 2014.

[17] D. Gfeller, A. Grosdidier, M. Wirth, A. Daina, O. Michielin, and V. Zoete, "Swisstargetprediction: a web server for target prediction of bioactive small molecules," Nucleic Acids Research, vol. 42, no. W1, pp. W32-W38, 2014.

[18] M. Kuhn, D. Szklarczyk, A. Franceschini, C. von Mering, L. J. Jensen, and P. Bork, "STITCH 3: zooming in on proteinchemical interactions," Nucleic Acids Research, vol. 40, no. D1, pp. D876-D880, 2012.

[19] The UniProt Consortium, "UniProt: the universal protein knowledgebase," Nucleic Acids Research, vol. 46, no. 5, pp. D158-D169, 2017.

[20] D. W. Huang, B. T. Sherman, and R. A. Lempicki, "Systematic and integrative analysis of large gene lists using DAVID bioinformatics resources," Nature Protocols, vol. 4, no. 1, pp. 44-57, 2009.

[21] K.-Y. Hsin, Y. Matsuoka, Y. Asai et al., "SystemsDock: a web server for network pharmacology-based prediction and analysis," Nucleic Acids Research, vol. 44, no. W1, pp. W507-W513, 2016.

[22] B. H. May, C. Lu, L. Bennett, H. M. Hügel, and C. C. L. Xue, "Evaluating the traditional Chinese literature for herbal formulae and individual herbs used for age-related dementia and memory impairment," Biogerontology, vol. 13, no. 3, pp. 299-312, 2012.

[23] H. Bahrani, J. Mohamad, M. Paydar, and H. Rothan, "Isolation and characterisation of acetylcholinesterase inhibitors from Aquilaria subintegra for the treatment of Alzheimer's disease (AD)," Current Alzheimer Research, vol. 11, no. 2, pp. 206-214, 2014.

[24] P. Babaei, A. Jafari, and S. Kouhestani, "Kaempferol attenuates cognitive deficit via regulating oxidative stress and neuroinflammation in an ovariectomized rat model of sporadic dementia," Neural Regeneration Research, vol. 13, no. 10, pp. 1827-1832, 2018.

[25] T. Beg, S. Jyoti, F. Naz et al., "Protective effect of kaempferol on the transgenic Drosophila model of Alzheimer's disease," CNS \& Neurological Disorders-Drug Targets, vol. 17, no. 6, pp. 421-429, 2018.

[26] S. P. Navabi, A. Sarkaki, E. Mansouri, M. Badavi, A. Ghadiri, and Y. Farbood, "The effects of betulinic acid on neurobehavioral activity, electrophysiology and histological changes in an animal model of the Alzheimer's disease," Behavioural Brain Research, vol. 337, pp. 99-106, 2018.

[27] A. Sarkaki, Y. Farbood, M. Badavi et al., "The protective effect of betulinic acid on microvascular responsivity and protein expression in Alzheimer disease induced by cerebral microinjection of beta-amyloid and streptozotocin," Microcirculation, vol. 25, no. 8, Article ID e12503, 2018.

[28] M. Ayaz, M. Junaid, F. Ullah et al., “Anti-Alzheimer's studies on beta-sitosterol isolated from polygonum hydropiper L," Frontiers in Pharmacology, vol. 8697 pages, 2017.
[29] S. Lee, K. Youn, and M. Jun, "Major compounds of red ginseng oil attenuate $\mathrm{A} \beta 25$-35-induced neuronal apoptosis and inflammation by modulating MAPK/NF- $\kappa \mathrm{B}$ pathway," Food \& Function, vol. 9, no. 8, pp. 4122-4134, 2018.

[30] S. Park, K. Sapkota, S. Kim, H. Kim, and S. Kim, "Kaempferol acts through mitogen-activated protein kinases and protein kinase B/AKT to elicit protection in a model of neuroinflammation in BV2 microglial cells," British Journal of Pharmacology, vol. 164, no. 3, pp. 1008-1025, 2011.

[31] C. Li, C. Zhang, H. Zhou et al., "Inhibitory effects of betulinic acid on LPS-induced neuroinflammation involve M2 microglial polarization via CaMKKbeta-dependent AMPK activation," Frontiers in Molecular Neuroscience, vol. 1198 pages, 2018.

[32] C. L. Masters, R Bateman, K Blennow, C. C Rowe, R. A Sperling, and J. L Cummings, "Alzheimer's disease," Nature Reviews Disease Primers, vol. 1, no. 1, p. 15056, 2015.

[33] V. Duronio, "The life of a cell: apoptosis regulation by the PI3K/PKB pathway," Biochemical Journal, vol. 415, no. 3, pp. 333-344, 2008.

[34] F. Guo, X. Liu, H. Cai, and W. Le, "Autophagy in neurodegenerative diseases: pathogenesis and therapy," Brain $\mathrm{Pa}$ thology, vol. 28, no. 1, pp. 3-13, 2018.

[35] J. Chu, J.-G. Li, N. E. Hoffman, M. Madesh, and D. Praticò, "Degradation of gamma secretase activating protein by the ubiquitin-proteasome pathway," Journal of Neurochemistry, vol. 133, no. 3, pp. 432-439, 2015.

[36] L. Hong, H.-C. Huang, and Z.-F. Jiang, "Relationship between amyloid-beta and the ubiquitin-proteasome system in Alzheimer's disease," Neurological Research, vol. 36, no. 3, pp. 276-282, 2014.

[37] F. Munari, A. Bortot, M. Assfalg, and M. D'Onofrio, “Alzheimer's disease-associated ubiquitin mutant $\mathrm{Ubb}+1$ : properties of the carboxy-terminal domain and its influence on biomolecular interactions," International Journal of Biological Macromolecules, vol. 108, pp. 24-31, 2018.

[38] M. Feld, M. C. Krawczyk, M. Sol Fustiñana et al., "Decrease of ERK/MAPK overactivation in prefrontal cortex reverses early memory deficit in a mouse model of Alzheimer's disease," Journal of Alzheimer's Disease, vol. 40, no. 1, pp. 69-82, 2014.

[39] Q. Zhou, M. Wang, Y. Du et al., "Inhibition of c-Jun $\mathrm{N}$-terminal kinase activation reverses Alzheimer disease phenotypes in APPswe/PS1dE9 mice," Annals of Neurology, vol. 77, no. 4, pp. 637-654, 2015.

[40] J. K. Lee and N.-J. Kim, "Recent advances in the inhibition of p38 MAPK as a potential strategy for the treatment of Alzheimer's disease," Molecules, vol. 22, no. 8, pp. 1287-1309, 2017.

[41] W.-L. Fang, D.-Q. Zhao, F. Wang et al., "Neurotropin alleviates hippocampal neuron damage through a HIF-1 $\alpha / \mathrm{MAPK}$ pathway," CNS Neuroscience \& Therapeutics, vol. 23, no. 5, pp. 428-437, 2017.

[42] M. F. Iulita, M. B. Bistué Millón, R. Pentz et al., "Differential deregulation of NGF and BDNF neurotrophins in a transgenic rat model of Alzheimer's disease," Neurobiology of Disease, vol. 108, pp. 307-323, 2017.

[43] Y. Du, H.-T. Wu, X.-Y. Qin et al., "Postmortem brain, cerebrospinal fluid, and blood neurotrophic factor levels in Alzheimer's disease: a systematic review and meta-analysis," Journal of Molecular Neuroscience, vol. 65, no. 3, pp. 289-300, 2018.

[44] S. H. Choi, E. Bylykbashi, Z. K. Chatila et al., "Combined adult neurogenesis and BDNF mimic exercise effects on cognition in an Alzheimer's mouse model," Science, vol. 361, no. 6406, 2018. 


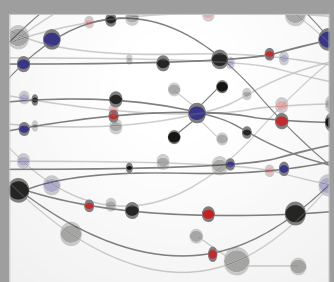

The Scientific World Journal
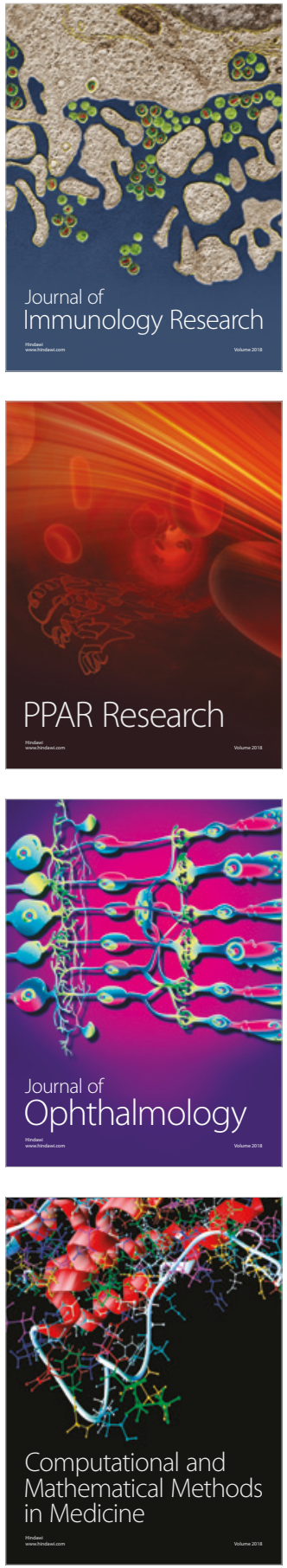

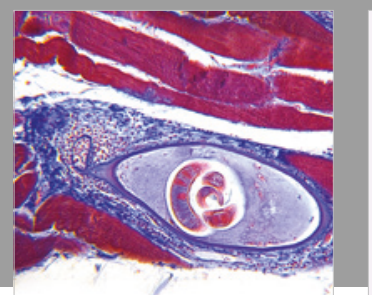

Gastroenterology Research and Practice

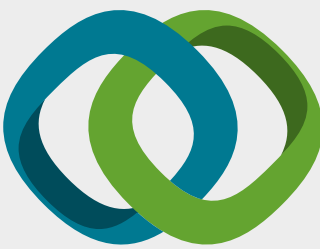

\section{Hindawi}

Submit your manuscripts at

www.hindawi.com
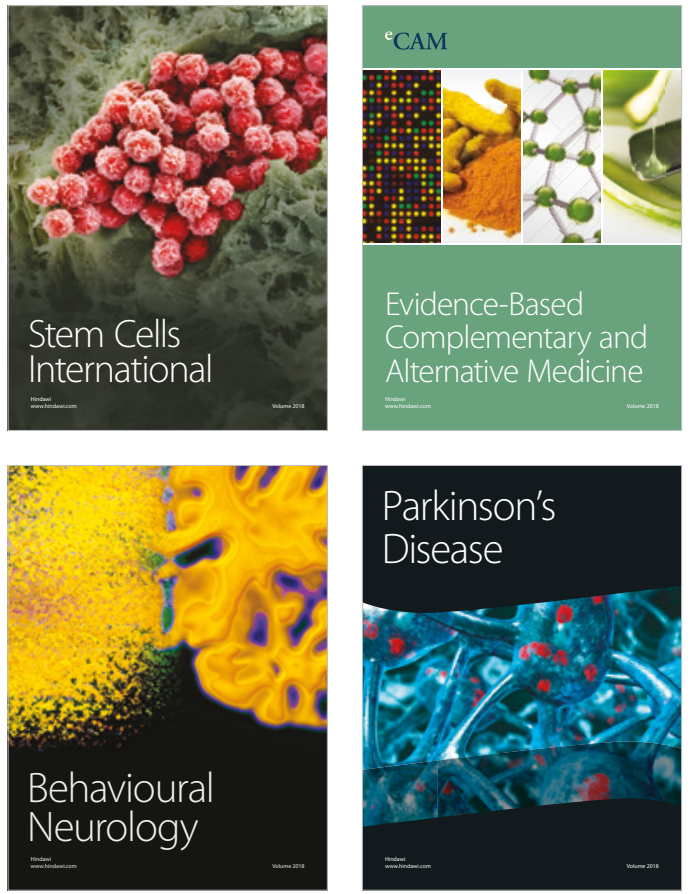

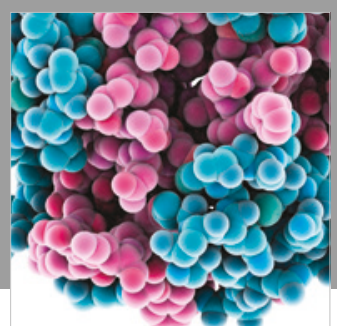

ournal of

Diabetes Research

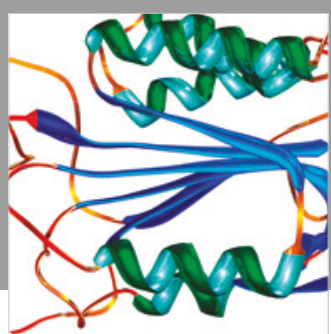

Disease Markers
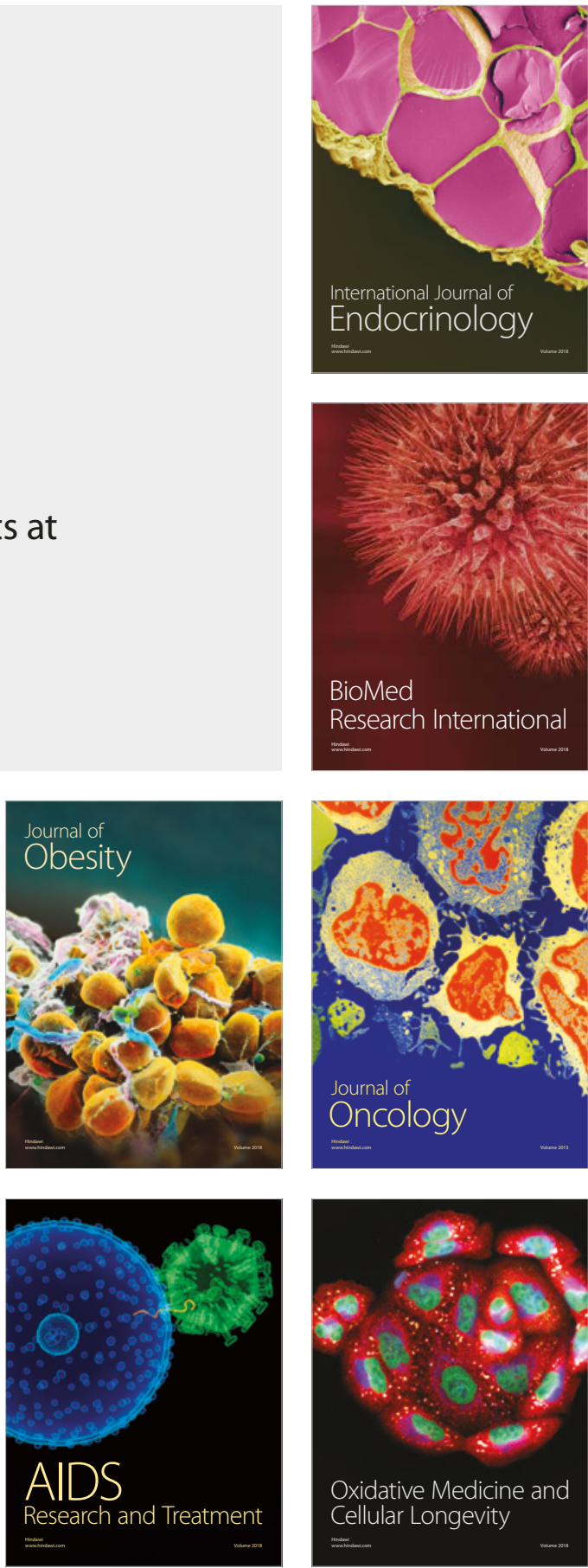